\title{
Medical Image of the Week: Paradoxical Stroke
}

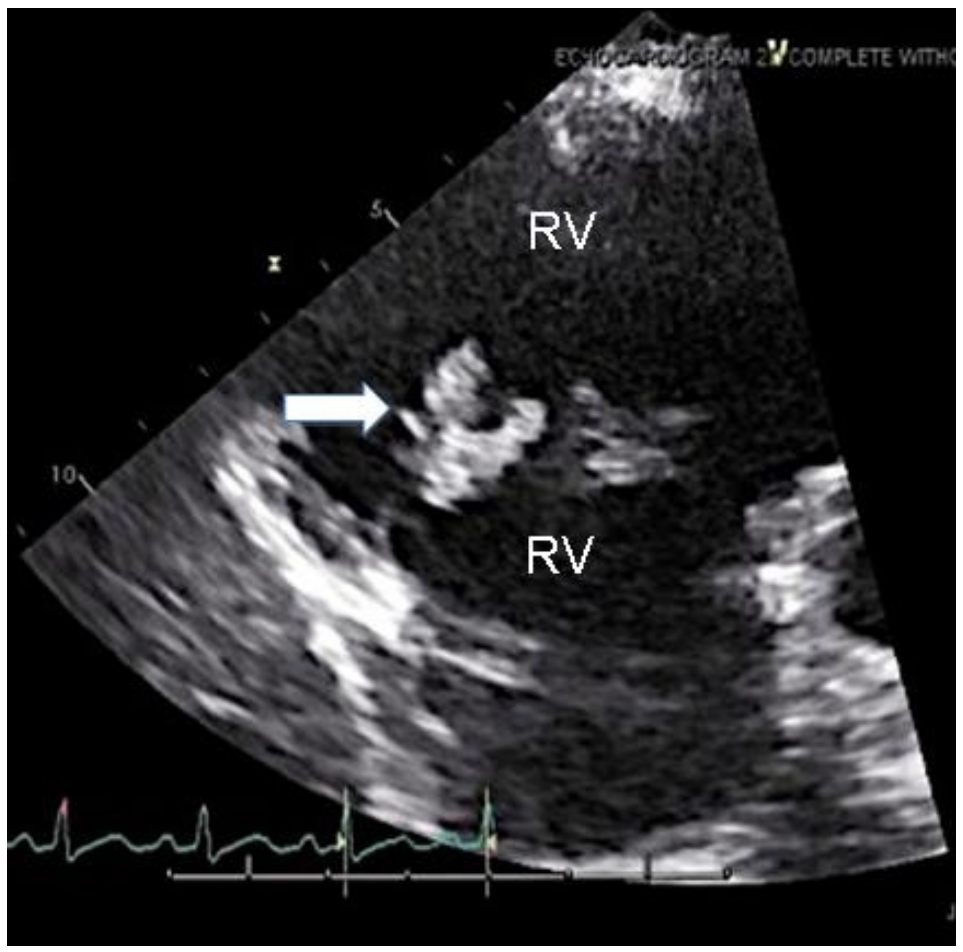

Figure 1. Vegetation seen on the tricuspid valve on the transthoracic echocardiogram (arrow). RA=right atrium, RV=right ventricle.

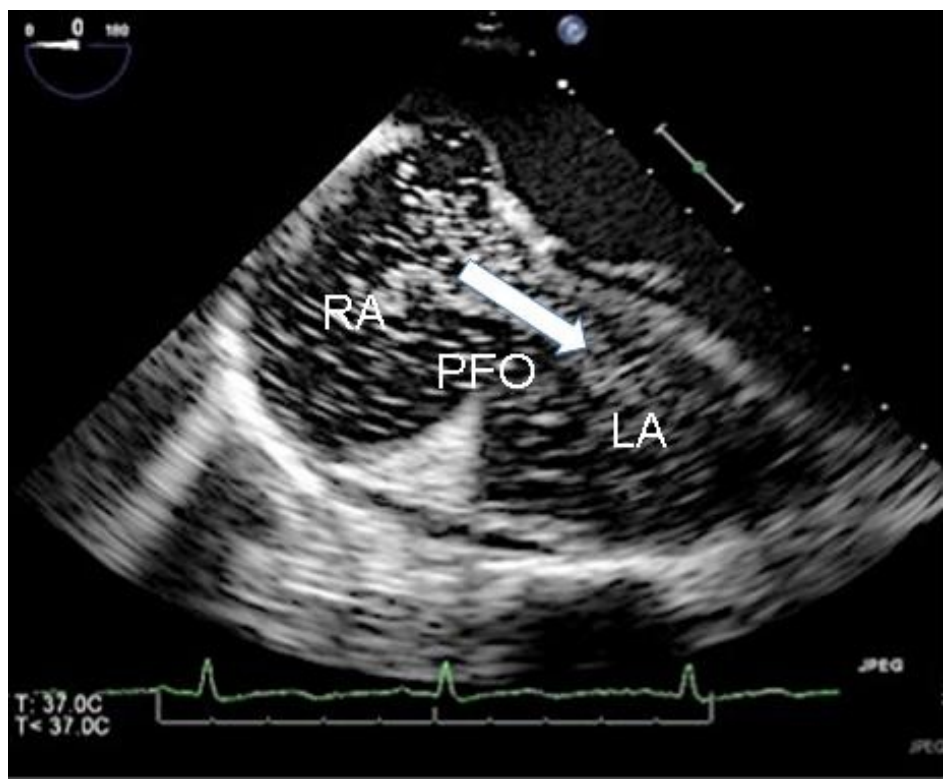

Figure 2. Patent foramen ovale (PFO) with right to left shunt of the agitated saline contrast on the trans-esophageal echocardiogram (arrow). RA=right atrium, $\mathrm{LA}=$ left atrium. 


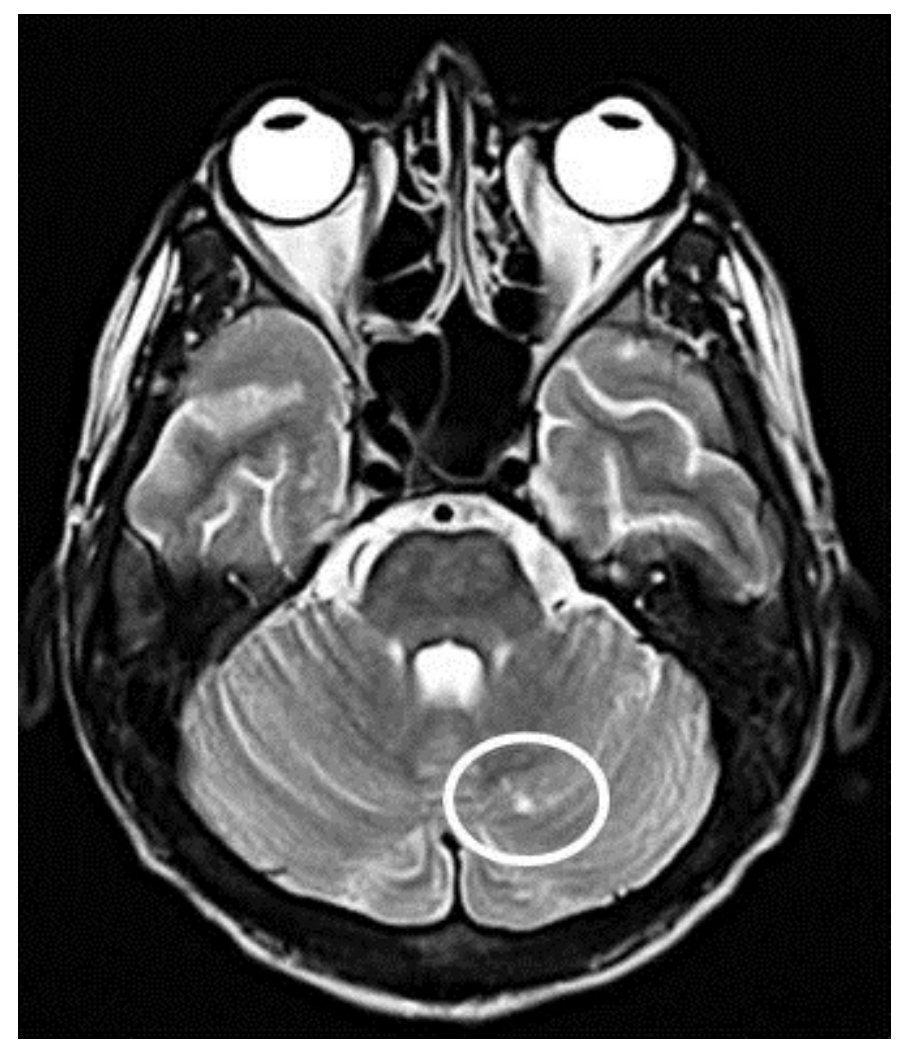

Figure 3. Acute left cerebellar stroke, hyper-dense lesion on T2 weighted MRI of the brain. (encircled).

A 23-year-old man with a history of intravenous drug abuse (IVDA) was admitted to the intensive care unit (ICU) secondary to sepsis. His blood cultures were positive for methicillin sensitive Staphylococcus aureus. Transthoracic echocardiogram showed vegetation on the tricuspid valve (Figure 1). He had multiple systemic emboli leading to suspicion for right to left shunt, which was confirmed by the agitated saline test during the echocardiogram (Figure 2). Cerebellar strokes likely secondary to posterior circulation embolic phenomenon was also seen (Figure 3). Overall, after a protracted ICU course complicated by multi-organ failure, he improved and is continuing treatment and rehabilitation at this time.

Right-sided infective endocarditis (IE) incidence is low, accounting for $5-10 \%$ of all cases of IE (1). IVDA is a well-known cause of tricuspid valve endocarditis. Usual features of tricuspid endocarditis are fever, bacteremia and pulmonary septic emboli. Patent foramen ovale (PFO) is estimated in up to $25 \%$ of the general population. Management of PFO for secondary stroke prevention remains controversial. Closure can be achieved surgically or percutaneously. The efficacy of closure of a PFO on the rate of recurrent stroke has not been established. 
Laila Abu Zaid $\mathrm{MD}^{1}$, Evbu Enakpene $\mathrm{MD}^{2}$ and Bhupinder Natt $\mathrm{MD}^{3}$

${ }^{1}$ Department of Internal Medicine

${ }^{2}$ Division of Cardiovascular Diseases

${ }^{3}$ Division of Pulmonary, Allergy, Critical Care and Sleep Medicine University of Arizona Medical Center

Tucson, AZ.

\section{Reference}

1. Akinosoglou K, Apostolakis E, Marangos M, Pasvol G. Native valve right sided infective endocarditis. Eur J Intern Med. 2013;24(6):510-9. [CrossRef] [PubMed] 